\title{
Tecnura
}

\section{Efectos de las crecidas en el transporte de material particulado y carbono orgánico. Caso del río Oka (País Vasco, España)}

\section{Effects of floods in the transport of particulate matter and organic carbon. Oka river case (Basque Country, Spain)}

\author{
Luis Hernando Montoya Armenta ${ }^{\circledR}$, Yim James Rodríguez Díaz ${ }^{\circledR}$
}

Fecha de recepción: 20 de Diciembre de 2019

Fecha de aceptación: 6 de Mayo de 2020

Cómo citar: Montoya-Armenta., L.H., y Rodríguez-Díaz., Y.J. (2020). Efectos de las crecidas en el transporte de material particulado y carbono orgánico. Caso del río Oka (País Vasco, España). Tecnura, 24(65) 64-76. DOI: $10.14483 / 22487638.15759$

\section{Resumen}

Objetivo: El objetivo general de esta investigación fue entender la repercusión de los eventos de crecidas en la exportación de sólidos suspendidos y carbono orgánico en la cuenca del río Oka, para el periodo comprendido desde 01-10-2009 a 30-09-2012.

Metodología: Se instaló una estación hidrometeorológica en un punto de la desembocadura de la cuenca, lo que permitió la recopilación continua de información concerniente a: pluviosidad, caudal del río, turbiedad, etc. La correlación entre la turbiedad medida en campo (TRB) y la concentración sólidos suspendidos (CSS), determinados a nivel de laboratorio fue establecida como una función polinómica de primer grado. La carga de sólidos suspendidos (SS), carbono orgánico en disolución (COD) y carbono orgánico en forma de partículas suspendidas o particulado (COP), fue determinada multiplicando el caudal por la concentración de cada uno de ellos, aplicándolo para cada periodo de tiempo requerido. Resultados: La exportación total se estimó en $2267,6 t$ de SS, $104,3 t$ de COD y $53,3 t$ de COP, representando tasas en $t \mathrm{~km}^{-2}$ año-1 de 71,85 para SS; 3,30 para COD, y 1,68 para COP (valores promedio de los tres años hidrológicos estudiados).

Conclusiones: El estudio estadístico reveló que, para esta cuenca, los agentes más trascendentes en el transporte de SS, COD y COP son la precipitación y el volumen de agua transportado. Los sólidos en suspensión se transportaron especialmente durante los meses comprendidos desde el otoño hasta el invierno, cuando se producen con frecuencia las crecidas más intensas, siendo estas las de mayor importancia en la contribución de sólidos suspendidos y carbono orgánico a la salida de la cuenca.

Palabras clave: carbono orgánico, crecidas, exportación, río Oka, sólidos en suspensión.

\footnotetext{
Abstract

Objective: The general objective of this research was to understand the repercussion of flood events on the export of suspended solids and organic carbon in the Oka river catchment for the period from 1001-2009 to 09-30-2012).
} 
Methodology: A hydrometeorological station was installed at a point at the catchment mouth allowing the continuous collection of information on: rainfaII, river flow, turbidity, etc. The correlation between turbidity measured in the field (TRB) and suspended solids concentration (CSS), determined at the laboratory level, was established as a polynomial function of the first degree. The load of suspended solids $(S S)$, organic carbon in dissolution (COD) and organic carbon in the form of suspended particles or particulate $(C O P)$, was determined by multiplying the flow rate by the concentration of each one, applying it for each period of time required.

Results: Total exports were estimated at 2,267.6 $t$ of $S S, 104.3 t$ of COD and $53.3 t$ of COP, representing rates in $t \mathrm{~km}^{-2}$ year-1 of 71.85 for SS, 3.30 for COD and 1.68 for $C O P$ (average values of the three hydrological years studied).

Conclusions: The statistical study revealed that for this catchment, the most important agents in the transport of $S S, C O D$ and $C O P$ are: precipitation and the volume of water transported. Suspended solids were transported especially during the months from autumn to winter, when the most intense floods occur most frequently, being these the most important in the contribution of suspended solids and organic carbon to the outlet of the catchment.

Financing: University of the Basque Country (UFI11/26). Keywords: Organic carbon, floods, exportation, Oka river, suspended solids.

\section{INTRODUCCIÓN}

En el interior de las cuencas hidrográficas, los recursos hídricos naturales, dispuestos principalmente para el consumo humano, están expuestos a una posible contaminación en el transcurso de su ciclo hidrológico. En estos se presentan significativos y súbitos incrementos de la concentración de ciertos contaminantes en la columna de agua, principalmente durante temporadas de avenidas, conocidas como crecidas o crecientes. Los contaminantes se asocian con la actividad antropogénica humana (agricultura, ganadería, industria, entre otras), y en ocasiones sus concentraciones pueden superar la capacidad autodepurativa de la naturaleza, afectando negativamente la calidad del recurso para sus posibles usos (Montoya, 2013).

Las crecidas presentan diversas variables hidrológicas, entre las más importantes están: el caudal, el volumen total de agua transportado o la velocidad con la cual se incrementa el caudal, las cuales se asocian con una precipitación equivalente en intensidad y duración; estas varían dependiendo de las particularidades de la cuenca, como tamaño, pendiente, tipo de suelo o características morfométricas; así mismo, por características alterables por actividades antropogénicas, como los usos del territorio, cubierta vegetal, zonas urbanas con superficies impermeables, etc. (Carvajal, Arango y Jiménez, 2007; Montoya, 2013; Zabaleta et al., 2007).

Por otra parte, la erosión del suelo genera un incremento en el arrastre y deposición de sedimentos y, por tanto, en la turbiedad de los ríos (González, Carvajal y Loaiza, 2016), puesto que el agua transportará el sedimento desde la parte alta de las cuencas hasta las zonas aluviales y de sedimentación, como son los estuarios y las desembocaduras al mar (Oeurng, Sauvage y Sánchez-Pérez, 2010; Montoya, 2013). La abundancia y características de las sustancias aportadas por las crecidas influyen sobre la calidad del agua en las zonas costeras de desembocadura, la preservación de los ecosistemas y sus posibles usos. Por otra parte, la exportación del sedimento en los ríos está asociado con la dinámica del transporte de carbono orgánico disuelto y particulado, sustancias de gran importancia para la calidad del recurso hídrico y que se utilizan como indicadores de contaminación (Ni et al., 2008; Montoya 2013).

Actualmente, la cuantificación de los sólidos suspendidos y el carbono orgánico exportados en las diferentes cuencas de los ríos ha adquirido 
relevancia para entender sus dinámicas de transferencia, lo cual es básico para la prevención de la erosión y los deslizamientos del suelo por escorrentía, especialmente en las laderas presentes en zonas de influencia en las cuencas de los ríos, lo que a su vez ha sido utilizado como herramienta para la gestión integrada de los recursos hídricos (Oeurng, Sauvage y Sánchez-Pérez, 2010; Oeurng et al., 2011; Montoya, Ruiz y Antigüedad, 2014). No obstante, son limitados los estudios que estimen las tasas de transporte de sedimento en suspensión y carbono orgánico en cuencas, principalmente durante eventos crecidas. Ejemplo de ello es la tesis doctoral "Efectos de las crecidas en el transporte de material particulado y contaminantes asociados: aplicación al caso del río Oka (Urdaibai), País Vasco" (Montoya, 2013), en la cual se basa este artículo. Parte de este trabajo fue presentado como ponencia en la I Jornada Binacional de Investigación de la URBE (Montoya, Ruiz y Antigüedad, 2014).

\section{METODOLOGÍA}

\section{Zona de estudio}

El área de estudio está ubicada en la unidad hidrográfica del Oka, este río realiza la más importante aportación de agua y material en suspensión desde el continente hasta su desembocadura en el estuario de Urdaibai (figura 1), el cual fue reconocido por la Unesco en 1984 como reserva de la biosfera de Urdaibai, por su extraordinaria abundancia ecológica (Montoya, 2013).

El río Oka recorre unos 14 km, y es catalogado como de corto tramo. La cuenca se sitúa en la provincia de Bizkaia (País Vasco), con una extensión de $178 \mathrm{~km}^{2}$. Este estudio se realizó en la subcuenca de cabecera del Oka $\left(31,56 \mathrm{~km}^{2}\right)$, siendo esta homónima con la cuenca principal y de carácter principalmente agroforestal, ostentando una pendiente promedio aproximada del 26 \% (Montoya, 2013).

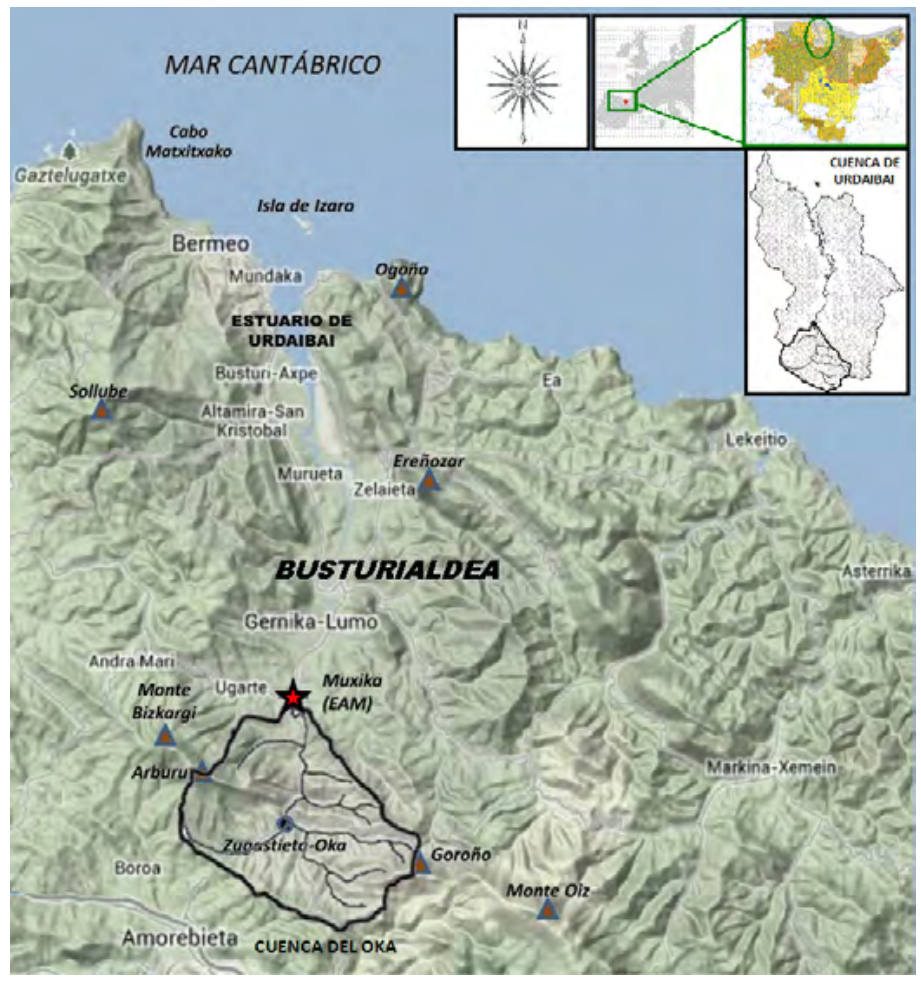

Figura 1. Mapa de localización de la parte alta de la cuenca del río Oka

Fuente: elaboración propia. 


\section{Instrumentación y métodos de muestreo}

La red hidrometeorológica de Bizkaia posee una estación de aforo emplazada en la población de Muxika (EAM) que permite monitorear continuamente variables como el caudal del río, precipitación, turbiedad, entre otras. Estas variables se registran electrónicamente en intervalos de diez minutos. La turbiedad se midió directamente en el cauce del río usando un turbidímetro infrarrojo Solitax con un rango de 0-1000 NTU. Para garantizar el monitoreo de los eventos de crecidas, en la estación de aforos se instaló un muestreador de agua automático (SIGMA 900) y se programó para iniciar la recolección de 24 muestras (cada una con intervalo de dos horas), cuando la turbiedad en la corriente alcanzara 100 NTU. Las muestras de agua de 25 eventos de crecida fueron recolectadas en botellas de polietileno de $800 \mathrm{ml}$ y transportadas inmediatamente al Laboratorio de Ingeniería Química y Ambiental de la Universidad del País Vasco, para su procesamiento y posterior análisis (Montoya, 2013).

\section{Análisis de laboratorio}

A las muestras tomadas durante los eventos de crecida (sin filtrar) se les corroboró el valor de turbiedad y se comparó con los datos medidos en el cauce del río. La concentración de sólidos suspendidos (CSS) se midió por filtración de la muestra bruta (300 a $400 \mathrm{ml}$ ) a través de un filtro previamente pesado de $0,45 \mu \mathrm{m}$, para ser subsecuentemente secado y pesado. El carbono orgánico total (COT) y el carbono orgánico disuelto (COD) se determinaron por oxidación con ácido crómico en una muestra de agua sin filtrar y filtrada, respectivamente, mediante un método colorimétrico (APHA, 2005). El carbono orgánico particulado se calculó por la diferencia entre el COT y COD (Montoya, 2013).

\section{Determinación de cargas y tasas de exportación}

Basándose en el registro de los datos de caudal y la concentración de sólidos en suspensión, carbono orgánico disuelto y particulado, determinados para intervalos de 10 minutos, se establecieron las cargas anuales de SS, COD y COP con el procedimiento planteado por Walling y Webb (1985), de acuerdo con la ecuación (1).

$$
\text { CARGA }=V * \frac{\sum_{i=1}^{n}\left(C_{i} * Q_{i}\right)}{\sum_{i=1}^{n} Q_{i}}
$$

Donde: $V\left(m^{3}\right)$ es el volumen de agua descargado durante el intervalo de tiempo requerido; $\mathrm{Ci}$ es el elemento concentración ( $m g L^{-1}$ para SS, COD y COP); Qi $\left(\mathrm{m}^{3} \mathrm{~s}^{-1}\right)$ es el caudal instantáneo del río y $n$ es el número de mediciones (Oeurng et al., 2011; Montoya, 2013). A partir del cociente realizado entre la carga anual y el área de la cuenca de cabecera $\left(31,56 \mathrm{~km}^{2}\right)$, se calcularon las tasas de exportación para SS, COD y COP $\left(t \mathrm{~km}^{-2}\right.$ año-1 $)$.

\section{Análisis estadístico}

El procesamiento estadístico de los datos se realizó mediante el software SPSS. Se efectuó un análisis de componentes principales (ACP) con rotación ortogonal Varimax para establecer relaciones entre variables y posibles patrones en la distribución de los datos medidos.

\section{RESULTADOS}

Las curvas ascendentes y descendentes de los hidrogramas representan los eventos hidrológicos de las crecidas (Oeurng et al., 2011). De acuerdo con esto, se realizó la caracterización de 25 eventos de crecida durante este estudio, acorde a las variables que han sido empleadas por diversos investigadores (Oeurng et al., 2011; Montoya, 2013; Cerro, 
Sánchez-Pérez, Ruiz-Romera y Antigüedad, 2014). Las variables se agruparon en aquellas que ofrecen información antes y durante los eventos de crecida, las cuales se resumen en la tabla 1.

Las variables antecedentes comprenden la precipitación acumulada de un día antes ( $P 1 d, \mathrm{~mm})$, siete días antes $(P 7 d, \mathrm{~mm})$ y veintiún días antes $(P 21 \mathrm{~d}, \mathrm{~mm})$ del inicio de la crecida, así como el caudal base también anterior al inicio de la crecida $\left(Q b, \mathrm{~m}^{3} \mathrm{~s}^{-1}\right)$. Las variables de la crecida comprenden la precipitación total causante de las crecidas $(P t, \mathrm{~mm})$, intensidad para la precipitación media por hora $\left(I P m, \mathrm{~mm} \mathrm{~h}^{-1}\right)$, máxima intensidad para una precipitación por hora (IPmáx, $\mathrm{mm} \mathrm{h}^{-1}$ ) durante el evento. Asimismo, se expresaron: el volumen agua total producida o volumen de la crecida $\left(V_{C^{\prime}} \mathrm{hm}^{3}\right)$, la duración total de la crecida $(F d$, h), el caudal medio $\left(Q m, \mathrm{~m}^{3} \mathrm{~s}^{-1}\right)$, el tiempo $(T r, \mathrm{~h})$ para alcanzar el caudal máximo de crecida (Qmáx, $\left.\mathrm{m}^{3} \mathrm{~s}^{-1}\right)$ y se contemplaron parámetros como el coeficiente de la crecida $R C=V_{C} / P t\left(V_{C}\right.$ y $P t$ en $\left.\mathrm{mm}\right)$ y la relación de caudales $Q m a ́ x / Q b$. Los resultados del porcentaje de aporte de agua de la crecida con respecto al volumen de agua exportada durante el respectivo año hidrológico también son incluidos en la tabla 1.

Tabla 1. Variables hidrometeorológicas de las crecidas en la cuenca del Oka durante el periodo 2009-2012

\begin{tabular}{|c|c|c|c|c|c|c|c|c|c|c|c|c|c|c|c|c|c|c|}
\hline "* & E & & tación & $\begin{array}{l}P_{t} \\
m\end{array}$ & $\begin{array}{l}\boldsymbol{F}_{\boldsymbol{d}} \\
(\boldsymbol{h})\end{array}$ & $\begin{array}{c}\boldsymbol{T}_{r} \\
(\boldsymbol{h})\end{array}$ & $\begin{array}{l}I^{\prime} P_{m} \\
n m h\end{array}$ & $\left.m m^{m a x} h^{-1}\right)$ & $\begin{array}{c}P 1 d \\
(\mathrm{~mm})\end{array}$ & & ) & $7^{\left.c^{3}\right)}$ & $\begin{array}{c}\% \\
V_{C} / V_{T}\end{array}$ & $\mathbf{R}_{c}$ & $Q_{m}$ & $\begin{array}{c}Q_{b} \\
\left(m^{3} s^{-1}\right)\end{array}$ & $x$ & $2_{\text {max }} / Q_{b}$ \\
\hline \multirow{6}{*}{ 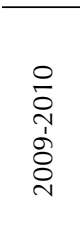 } & 1 & $/ 11$ & & 2,3 & 3,67 & 109,6 & 79 & 0 & 0,0 & & 7 & 4,64 & & & 4 & 12 & 93 & 4 \\
\hline & 2 & & & & & & & & & & & & & & & & & \\
\hline & 3 & 10 & & & & & & & & & & & & & & 47 & & \\
\hline & 4 & & & & & & & & & & & & & & & & & \\
\hline & 5 & & & & & & & & & & & & & & & & & \\
\hline & 6 & & & 3 & 0 & 9,00 & & & & 6 & & 1 & & & & & 31 & \\
\hline \multirow{10}{*}{$\begin{array}{l}\bar{\sigma} \\
\stackrel{\sim}{1} \\
\text { o } \\
\frac{1}{0}\end{array}$} & 7 & & & & & & & & & & & & & & & & & \\
\hline & 8 & & & & & & & & & & & & & & & & & \\
\hline & 9 & & & 1 & 0 & & & & & 4 & & & & & & & & \\
\hline & 10 & & & & 12,33 & U & & & & 52,1 & & 1, & & & 2,87 & & & 05 \\
\hline & 11 & & & & & & & & & & & & & & & & & \\
\hline & 12 & & & & & & & & & & & & & & & & & \\
\hline & 13 & & & 0 & 83 & 2 & & & & 99,3 & 12 & & & & 2,41 & & 94 & 02 \\
\hline & 14 & & & & & & & & & & & & & & 6,43 & & & 1,68 \\
\hline & 15 & & & & & & & & & & & & & & & & & \\
\hline & 16 & & & & & & & & & 45 & & & & & & & & 76 \\
\hline \multirow{9}{*}{ 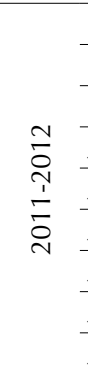 } & 17 & & & & & & & & & 7 & & & & & 4,70 & & & 1,23 \\
\hline & 18 & & & & & & & & & & & & & & & & & \\
\hline & 19 & & & & & & & & & 28,0 & & & & & & & & \\
\hline & 20 & & 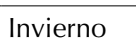 & 25,0 & 0 & 13,17 & 0,36 & 0 & & 28,4 & 47,6 & 0,59 & 2, & & 2,36 & & 5 & 38 \\
\hline & 21 & & & ,7 & 7 & 8,67 & 7 & & & 34,8 & & 0 & & & 41 & & & 31 \\
\hline & 22 & & & 9,8 & & J & & & & 29,6 & & & & & 3,00 & & & 5,54 \\
\hline & 23 & & & 1, & & 39, & & & & 85,8 & & & & & 2,38 & & & \\
\hline & 24 & & & 3,5 & & & & & & 5,4 & & & & & 2,63 & & 25 &, 39 \\
\hline & 25 & $20 / 05 / 2012$ & Primavera & 65,8 & 95,67 & 22,67 & 0,69 & 6,70 & 10,3 & 10,3 & 21,1 & 1,00 & 4,89 & 0,48 & 2,90 & 0,26 & 8,00 & 30,39 \\
\hline \multicolumn{19}{|c|}{$\begin{array}{l}E \text { : evento, } P_{t}: \text { precipitación total durante la crecida; } F_{d} \text { : duración de la crecida; } T_{r} \text { : tiempo para alcanzar la máxima descarga; } I P_{m} \text { : } \\
\text { intensidad de precipitación media; } I P_{\text {máx }}: \text { intensidad de precipitación máxima; } P 1 d, P 7 d, P 21 d \text { : precipitación acumulada un día, } \\
\text { siete días y veintiún días antes del evento; } V_{c}: \text { volumen de la crecida; } \% V_{c} V_{T}: \text { porcentaje de aporte de agua de la crecida con } \\
\text { respecto al volumen de agua exportada durante el respectivo año hidrológico; } R_{c}: \text { coeficiente de la crecida; } Q_{m}: \text { caudal medio; } \\
Q_{b}: \text { caudal base; } Q_{\text {máx }}: \text { caudal máximo; } Q_{\text {máx }} / Q_{b}: \text { relación caudal máximo/caudal base. }\end{array}$} \\
\hline
\end{tabular}

Fuente: elaboración propia. 
El $36 \%$ de estas crecidas (eventos 1, 2, 7, 8, $9,10,11,17$ y 18 ) ocurrieron en otoño (octubre a diciembre), el $32 \%$ (eventos 3, 4, 12, 13, 14, 19, 20 y 21 ) en invierno (enero a marzo), $24 \%$ (eventos $5,6,22,23,24$ y 25) en primavera (marzo a junio) y $8 \%$ (eventos 15 y 16) en verano (julio a septiembre). La precipitación para las 25 crecidas presentó un valor promedio de $66,5 \mathrm{~mm}$ y un rango que abarca desde 14,4 mm (julio 2011) hasta $262,3 \mathrm{~mm}$ (noviembre 2009), fechas que para la duración total del evento se corresponden con el mínimo (30,5 h) y máximo $(353,7$ h), con 99,8 h en promedio. Por otra parte, el volumen de agua desplazado durante los eventos presentó un promedio de $1,14 \mathrm{hm}^{3}$ con un mínimo de $0,07 \mathrm{hm}^{3}$ (julio 2011) y un máximo de $4,64 \mathrm{hm}^{3}$ (noviembre 2009). El caudal máximo registrado para los eventos presentó un rango comprendido entre $0,88 \mathrm{~m}^{3} \mathrm{~s}^{-1}$ (julio 2011) y 58,86 $\mathrm{m}^{3} \mathrm{~s}^{-1}$, este último durante el evento 14 (marzo 2011), con promedio de 14,34 $\mathrm{m}^{3} \mathrm{~s}^{-1}$ (Montoya, 2013).

De la tabla 1, también puede observarse que existen cuatro eventos de crecida que resaltan por su magnitud (eventos 1, 6, 14 y 17), los cuales pueden considerarse de alta intensidad. Estos presentaron los mayores caudales punta debido a altas precipitaciones generadas. Sin embargo, crecidas como la ocurrida en marzo 16 de 2011 no mostraron precipitaciones ni un tiempo de duración superior a algunas otras que se generaron, pero sí ostentó el máximo caudal registrado $\left(58,86 \mathrm{~m}^{3} \mathrm{~s}^{-1}\right)$, esto fue debido a que la intensidad de precipitación por hora fue alta dentro de las cuatro horas anteriores al pico de caudal, periodo en el cual se presentó la máxima intensidad de precipitación $\left(11,8 \mathrm{~mm} \mathrm{~h}^{-1}\right)$.

La concentración de sólidos suspendidos (CSS) promedio para las crecidas presentó un rango de valores comprendido desde 32,90 $\mathrm{mg} \mathrm{L}^{-1}$ (diciembre 2010) hasta 203,48 $\mathrm{mg} \mathrm{L}^{-1}$ (junio 2010). Entre tanto, la CSS máxima que se alcanzó fue de 1407,66 mg L-1 en el evento 14 (marzo 2011), donde también se presentaron valores punta para
$\operatorname{COD}\left(10,68 \mathrm{mg} \mathrm{L}^{-1}\right)$ y $\operatorname{COP}\left(21,34 \mathrm{mg} \mathrm{L}^{-1}\right)$, hecho en parte previsible por ser la crecida con el mayor caudal alcanzado, lo cual contribuiría a que el arrastre de sedimentos y carbono orgánico fuera el más significativo. De igual forma, se estableció que durante los eventos de crecida COD y COP hubo concentraciones promedio de 5,19 $\mathrm{mg} \mathrm{L}^{-1} \mathrm{y}$ $3,41 \mathrm{mg} \mathrm{L}^{-1}$, respectivamente, mientras que durante los periodos de estiaje se observó un valor promedio de 2,13 $\mathrm{mg} \mathrm{L}^{-1}$ para COD y $1,14 \mathrm{mg} \mathrm{L}^{-1}$ para COP (Montoya, 2013).

Los datos continuos de CSS durante todo el período de estudio (2009-2012) se calcularon a partir de una buena relación entre la turbiedad (TRB) medida en campo y la CSS obtenida en el laboratorio (figura 2). Esta regresión lineal $\left(R^{2}=0,94\right)$ indica que las propiedades físicas del material particulado en suspensión tienden a ser estables, señalando que no se presentan variaciones importantes en la fuente de los sedimentos debido a la homogeneidad de la cuenca en términos del tipo y uso del suelo (Walling y Webb, 1985).

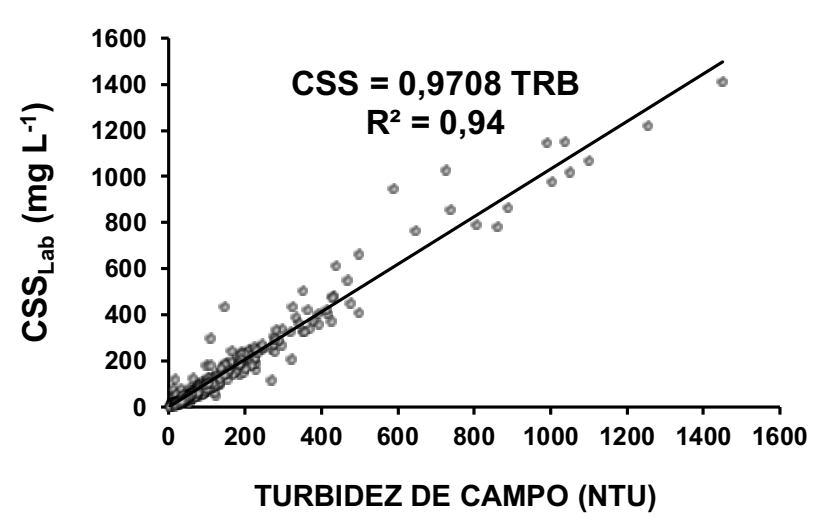

Figura 2. Regresión TRB - CSS para la cuenca del río Oka Fuente: elaboración propia.

Para obtener datos continuos de la concentración de COD y COP, se establecieron ecuaciones de regresión con el caudal durante los eventos de crecida $\left(R^{2}=0,61\right)$ y con CSS $\left(R^{2}=0,76\right)$, respectivamente (figura 3 ). 

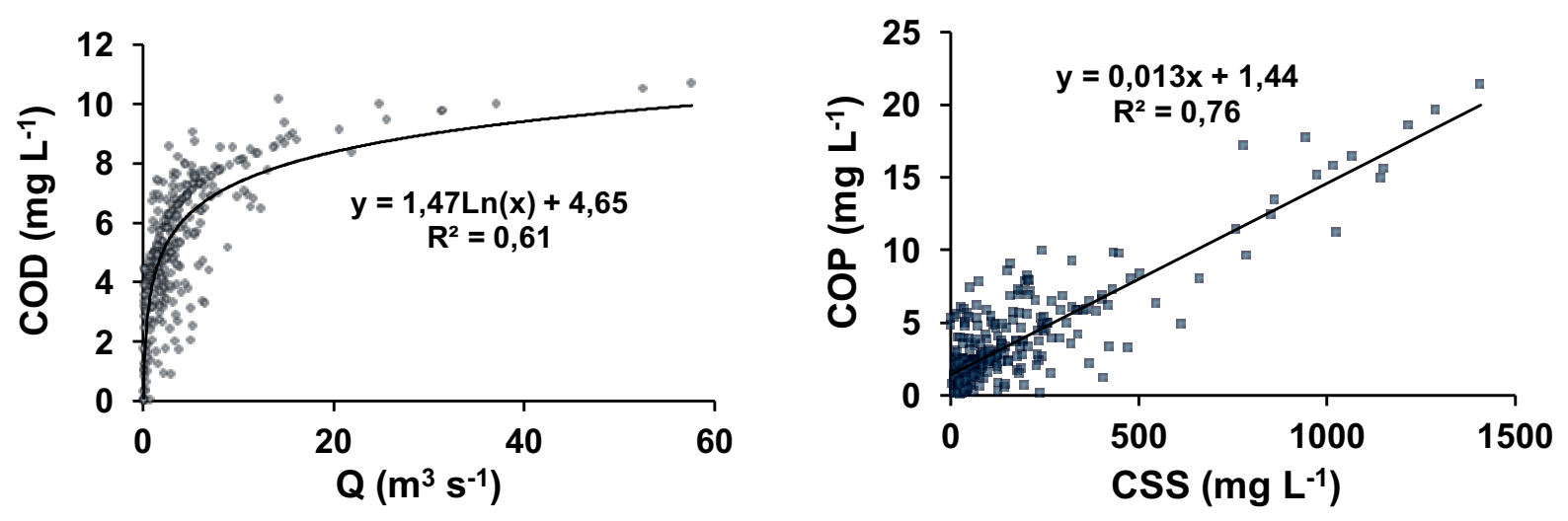

Figura 3. Relaciones entre (a) caudal $(Q)$ y carbono orgánico disuelto (COD); (b) concentración de sólidos suspendidos (CSS) y carbono orgánico particulado (COP)

Fuente: elaboración propia.

\section{Estimación de cargas y tasas específicas de exportación}

La tabla 2 resume las cargas medias y tasas específicas por año de SS, COD y COP para la cuenca de cabecera del río Oka. Durante el periodo hidrológico estudiado comprendido desde octubre de 2009 a septiembre de 2010, se exportó una carga total de $S S$ de 2777,63 toneladas (t), equivalente a $88,01 \mathrm{t} \mathrm{km}^{-2}$ año ${ }^{-1}$ (tasa específica); igualmente, se determinaron las cargas de COD $(112,58 \mathrm{t})$ y COP $(61,31 \mathrm{t})$ las cuales se corresponden con una tasa de $3,57 \mathrm{t} \mathrm{km}^{-2}$ año ${ }^{-1}$ para COD y 1,94 t km año $^{-1}$ para COP. En el periodo hidrológico comprendido desde 2010 a 2011, la carga media anual de SS, COD y COP se estimó en 2072,67 t, 90,98 t y $48,29 \mathrm{t}$, respectivamente, correspondiéndose con tasas de $65,67,2,88$ y $1,53 \mathrm{t} \mathrm{km}^{-2}$ año-1, respectivamente. El tercer periodo hidrológico estudiado (2011 a 2012) presentó cargas de exportación de 1952,19 t, 109,15 t y 49,94 t, para SS, COD y COP, respectivamente, con sus correspondientes tasas precisadas en $61,86,3,46$ y $1,58 \mathrm{t} \mathrm{km}^{-2}$ año $^{-1}$, respectivamente (Montoya, 2013).

En general, el periodo hidrológico 2009 a 2010 presentó una mayor pluviosidad, lo cual generó un mayor transporte de SS, COD y COP, si se compara con los dos periodos hidrológicos siguientes. El descenso que puede observarse en el transporte de sedimentos (25,38\% para 2010 a 2011 , y $29,72 \%$ para 2011 a 2012) refleja el vínculo directo entre la pluviosidad y la exportación de sedimentos. No obstante, se observó que la exportación de COD y COP no mantiene específicamente esta

Tabla 2. Valores generales y tasas específicas por año de SS, COD y COP transportados en la cuenca del río Oka

\begin{tabular}{|c|c|c|c|c|c|c|c|}
\hline $\begin{array}{c}\text { Año } \\
\text { hidrológico }\end{array}$ & $\begin{array}{c}\mathbf{P}_{\mathrm{T}} \\
(\mathbf{m m})\end{array}$ & $\begin{array}{c}\text { SSt } \\
\left(\mathrm{t} \mathbf{a n ̃ o}^{-1}\right)\end{array}$ & $\begin{array}{c}\text { SSt } \\
\left(\mathrm{t} \mathrm{km}^{-2} \text { año-1 }\right)\end{array}$ & $\left.\begin{array}{c}\text { CODt } \\
(\mathrm{t} \text { año } \\
-1\end{array}\right)$ & $\begin{array}{c}\text { CODt } \\
\left(\mathrm{t} \mathrm{km}^{-2} \text { año-1 }{ }^{-1}\right)\end{array}$ & $\left.\begin{array}{c}\text { COPt } \\
(t \text { año } \\
-1\end{array}\right)$ & $\begin{array}{c}\text { COPt } \\
\left(t \mathrm{~km}^{-2} \text { año-1 }\right)\end{array}$ \\
\hline $2009-2010$ & 1224,1 & 2777,63 & 88,01 & 112,58 & 3,57 & 61,31 & 1,94 \\
\hline $2010-2011$ & 1171,9 & 2072,67 & 65,67 & 90,98 & 2,88 & 48,26 & 1,53 \\
\hline 2011-2012 & 1071,6 & 1952,19 & 61,86 & 109,15 & 3,46 & 49,94 & 1,58 \\
\hline 2009-2012 & 1155,9 & 2267,50 & 71,85 & 104,24 & 3,30 & 53,17 & 1,68 \\
\hline
\end{tabular}

Fuente: elaboración propia. 
relación, lo cual estaría vinculado con las diversas particularidades de cada evento de crecida (tiempos y duración de los eventos, intensidad, volúmenes de agua exportados, etc.), al igual que con factores concernientes a las características y usos antrópicos del suelo, que pudieron presentarse en la cuenca en el periodo comprendido por este estudio y que podrían repercutir en que la variabilidad anual sea significativa (Oeurng, Sauvage y Sánchez-Pérez, 2010).

Para la cuenca del río Oka se estimó una tasa de exportación de sólidos suspendidos (sedimento en suspensión) correspondiente a 71,85 t km-2 año-1 como valor medio del periodo en estudio (octubre de 2009 a septiembre de 2012), la cual es menor al valor de 100 - $250 \mathrm{t} \mathrm{km}^{-2}$ año ${ }^{-1}$ publicado para cuencas perteneciente a la zona del mediterráneo español (Walling y Webb, 1996). De manera análoga se establecieron tasas de 3,30 $\mathrm{t} \mathrm{km}^{-2}$ año ${ }^{-1}$ para la exportación del carbono orgánico disuelto y 1,68 t km² $\mathrm{año}^{-1}$ para el carbono orgánico particulado.

En cuanto a las principales cuencas del País Vasco, existen claras diferencias entre unas y otras, dependiendo de su extensión y topografía. Uriarte (1998) realizó un estudio en la provincia de Gipuzkoa y reportó tasas de exportación de sedimento menores a las de este estudio, con valores de 45, 55 y $50 \mathrm{t} \mathrm{km}^{-2}$ año ${ }^{-1}$ en la cuenca de los ríos Deba $\left(450 \mathrm{~km}^{2}\right)$, Urola $\left(280 \mathrm{~km}^{2}\right)$ y Urumea $\left(210 \mathrm{~km}^{2}\right)$, respectivamente. En cambio, las cuencas de los ríos Oiartzun y Oria ostentaron tasas de exportación superiores, con 130 y 260 t km$^{-2}$ año $^{-1}$, respectivamente. Por otra parte, Zabaleta et al. (2007) analizaron la respuesta hidrosedimentaria en pequeñas cuencas de Gipuzkoa (País Vasco) durante los años hidrológicos 2003-2005, y estimó tasas de exportación de sedimentos de 45, 35 y $15 \mathrm{t} \mathrm{km}^{-2}$ año-1, respectivamente, para la cuenca de los ríos Añarbe $\left(48 \mathrm{~km}^{2}\right)$, Aixola $\left(4,8 \mathrm{~km}^{2}\right)$ y Barrendiola $\left(3 \mathrm{~km}^{2}\right)$. Estos autores también resaltan que resulta difícil establecer una comparación adecuada entre los resultados obtenidos para las distintas cuencas, ya que existen importantes diferencias entre las metodologías aplicadas por elaboración propia y las características de las cuencas (geología, relieve, precipitación, uso de suelo, entre otras).

Datos más recientes para estas y otras cuencas durante el periodo 2006-2012 han sido reportados por la Diputación Foral de Gipuzkoa en el informe Cuantificación de la tasa de sedimentos exportados de las cuencas del territorio histórico de Gipuzkoa (DFG, 2012). Entre ellas se destacan las cuencas de los ríos: Aixola (18 t km ${ }^{-2}$ año $\left.^{-1}\right)$, Añarbe (170 t km² año-1), Deba (60-105 t km² año-1), Urola (31-100 t km² año-1), Oria (171-350 t km² año-1), Urumea (46-127 $\left.\mathrm{t} \mathrm{km}^{-2} \mathrm{año}^{-1}\right)$, Oiartzun (52$115 \mathrm{t} \mathrm{km}^{-2}$ año ${ }^{-1}$ ) y Amundarain (38-52 t km² año-1). Este informe ha demostrado, además, la importancia del seguimiento permanente de variables físicas, químicas, meteorológicas e hidrológicas para dimensionar la magnitud y las variaciones con la que se exportan los sedimentos en las principales cuencas de Gipuzkoa.

La tasa media de COP $\left(1,68 \mathrm{t} \mathrm{km}^{-2}\right.$ año-1 $)$ determinada para la cuenca del río Oka durante todo el periodo en estudio, es ligeramente superior a la tasa de 1,47 t km-2 año-1 estimada por Veyssy et al. (1998) en la cuenca del Garona al suroeste de Francia, la tasa de 1,64 t km-2 año-1 en el río Orinoco (Ludwig, Probst y Kempe, 1996) y la tasa de 1,10 $\mathrm{t} \mathrm{km}^{-2}$ año ${ }^{-1}$ estimada como la media de los ríos en Europa (Ludwig, Probst y Kempe, 1996). Sin embargo, esta tasa es muy superior al valor hallado de 0,54 t km año $^{-1}$, reportado en la cuenca forestal Alegría ubicada en la provincia de Álava, al norte de España (Cerro et al., 2014). En contraste, esta tasa en el río Oka es inferior a la encontrada por Oeurng et al. (2011) en la cuenca Save al suroeste de Francia $\left(1,8 \mathrm{t} \mathrm{km}^{-2}\right.$ año-1 $)$, también a la estimada en el río Amazonas 3,23 t km² año-1 (Richey, Hedges, Devol y Quay, 1990) y mucho más baja que las 5,3 $\mathrm{t} \mathrm{km}^{-2}$ año ${ }^{-1}$ del río Nivelle que drena en una típica cuenca montañosa del Pirineo Francés hacia el Golfo de Vizcaya (Coynel et al., 2005).

En cuanto a la tasa media anual de COD $\left(1,68 \mathrm{t} \mathrm{km}^{-2}\right.$ año-1), este valor es superior a las estimaciones de $0,70 \mathrm{t} \mathrm{km}^{-2}$ año ${ }^{-1}$ para la cuenca del río Save (Oeurng et al., 2011), las 0,85 t km-2 año-1 
en la cuenca Alegría (Cerro et al., 2014), y 0,95 t km-2 año ${ }^{-1}$ estimadas en la región agrícola del río Embarras como el promedio de los años 1994 a 2003 (Royer y David, 2005). Sin embargo, este valor es inferior a otros encontrados en ríos suramericanos como el Amazonas con 5,27 t km-2 año-1 (Richey et al., 1990) y Orinoco con 4,82 t km² año-1 (Ludwig, Probst y Kempe, 1996). La obtención de una mayor carga de COD respecto al COP se relaciona principalmente con una limitada erosión del suelo presentada por las cuencas forestales a diferencia de las cuencas agrícolas y de uso mixto. Asimismo, los suelos en la cuenca del Oka, que están en su mayoría cubiertos de bosques y pastos, le proporcionarían a la corriente de agua un alto contenido de COD producto del material vegetal en descomposición (Yang et al., 2013).

\section{Variabilidad temporal de la exportación de SS, COD y COP}

La cuenca de cabecera del río Oka presentó, durante los tres años hidrológicos estudiados, una gran variabilidad temporal respecto a la exportación de sólidos en suspensión y carbono orgánico. Estos cambios estacionales pueden analizarse considerando las variaciones en el clima (Oeurng, Sauvage y Sánchez-Pérez, 2010; Cerro et al., 2014).

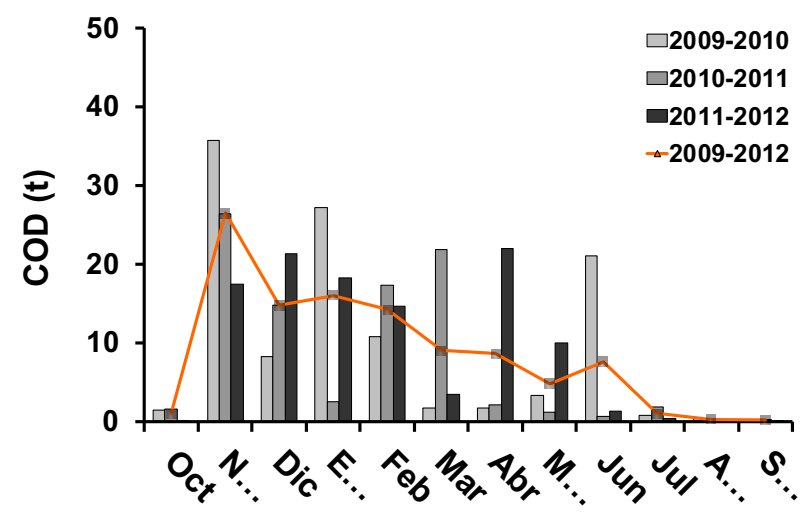

La contribución mensual de la carga de SS por cada año hidrológico analizado en la cuenca puede apreciarse en la figura 4; la línea naranja representa el valor promedio del mes, que se ha determinado teniendo en cuenta la información recopilada en cada uno de los periodos estudiados (Montoya, 2013).

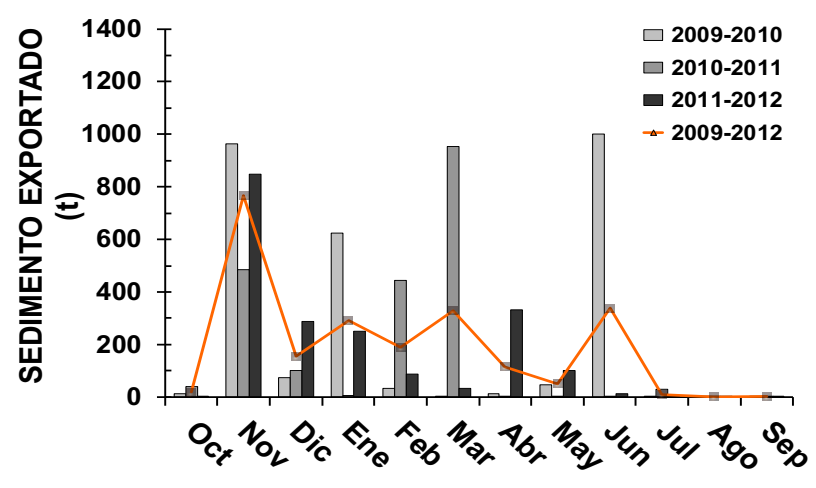

Figura 4. Cargas mensuales de SS exportados por la cuenca del río Oka en el periodo 2009-2012

Fuente: elaboración propia.

Particularmente, los principales aportes de SS, COD y COP tuvieron lugar desde el mes de noviembre hasta marzo (otoño/invierno), estaciones donde se originaron con una mayor frecuencia los eventos de lluvia intensa y continua (figura 5).

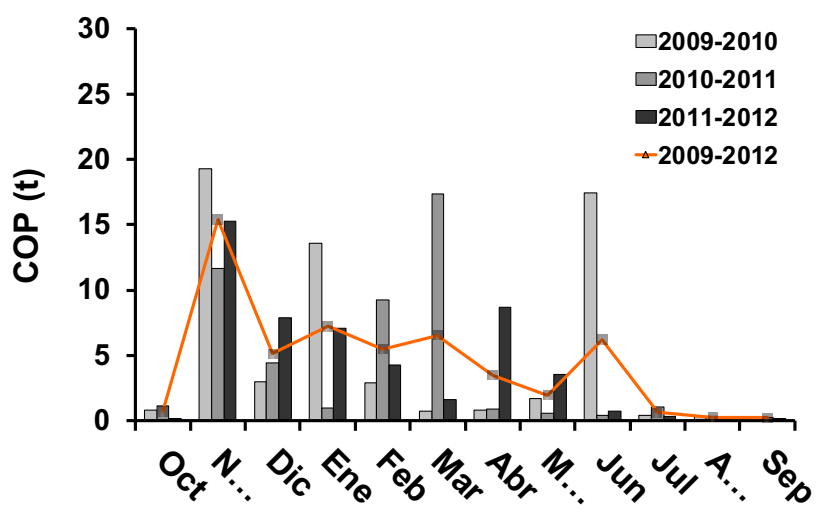

Figura 5. Cargas mensuales de: (a) COD; (b) COP, exportados por la cuenca del río Oka en el periodo 2009-2012 Fuente: elaboración propia. 
No obstante, el transporte de materiales por el río igualmente está supeditado al origen y características de crecidas inusitadas y poderosas que podrían suceder especialmente en otoño, invierno e, incluso, en primavera. De la misma manera, adquiere gran importancia la disponibilidad del sedimento y demás materiales en el propio cauce y las zonas aledañas al río, sufriendo procesos de arrastre o reposición a causa de las dinámicas de las corrientes de agua en la cuenca (Montoya, 2013).

\section{Relación entre las variables hidrometeorológicas, sólidos en suspensión y carbono orgánico}

Se obtuvieron cuatro factores que explican en total el 82,27 \% de la varianza, mediante el ACP. La figura 6 agrupa los factores más importantes (I y II), los cuales representan el 58,90 \% de la varianza total. En el primer factor se agrupan las variables $I P_{m^{\prime}} I P_{\text {máx }^{\prime}} Q_{m^{\prime}} Q_{\text {máx' }^{\prime}} C S S_{m^{\prime}} C S S_{\text {máx }^{\prime}} S S_{t^{\prime}} C O D_{\text {máx }^{\prime}}$ $C O P m, C O P_{\text {máx }} C^{\prime} P_{t}$ y en menor grado $C O D_{m^{\prime}}$ $Q_{\text {máx }} / Q_{b^{\prime}}$ que explican el $36,90 \%$ de su varianza. El segundo factor agrupa $P_{t^{\prime}} F_{d^{\prime}} T_{r^{\prime}} V_{c}$ y $C O D_{t^{\prime}}$ que explica el 22,00 \% de la varianza total. Las variables relacionadas con el sedimento en suspensión

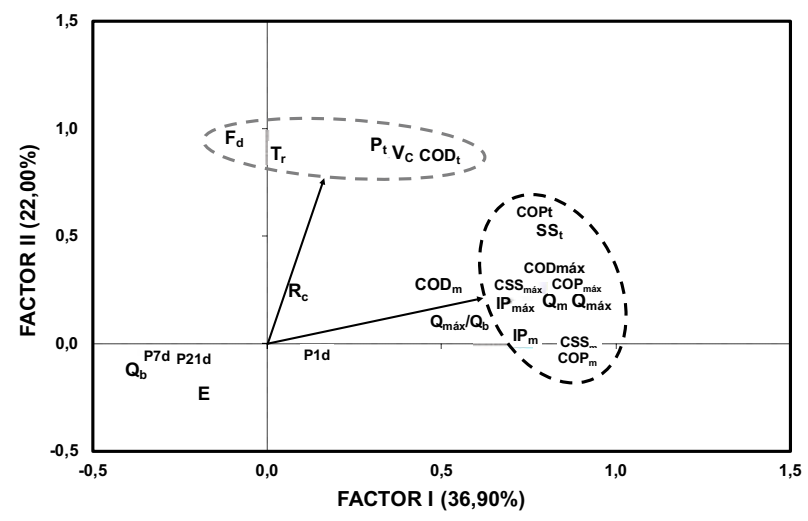

Figura 6. Ubicación de las variables hidrometeorológicas, SS, COD y COP, en los planos factoriales I - II del análisis de componentes principales

Fuente: elaboración propia. presentaron una fuerte relación con los factores I-II, pero principalmente con el primero. Estos resultados sugieren una respuesta directa en la cuenca en términos del caudal del río, la exportación de sedimento en suspensión y carbono orgánico ante los eventos con mayor importancia en términos de duración, intensidad de las precipitaciones y caudal máximo generado. En contraste, también se identifican parámetros de las crecidas $\left(E, Q_{b^{\prime}}\right.$ P1d, P7d, P21d y $R_{c^{\prime}}$ que no presentan influencia directa sobre la generación de sedimentos.

Igualmente, en la figura 7 se observa la distribución de las crecidas entre los factores I y II que se obtuvieron del ACP, identificando numéricamente cada uno de los eventos de acuerdo con la tabla 1.

Teniendo en cuenta estos componentes, la clasificación de los eventos de crecida se definió en tres categorías de acuerdo con su capacidad para transportar sólidos en suspensión y carbono orgánico.

Tipo I: son aquellas crecidas que presentan una menor intensidad y un alto porcentaje de ocurrencia en esta cuenca (48\%), representando doce eventos de crecida $(2,5,7,11,13,15,16,20,21$, 23, 24 y 25). Estas se corresponden con situaciones de bajas contribución de SS (<100 toneladas)

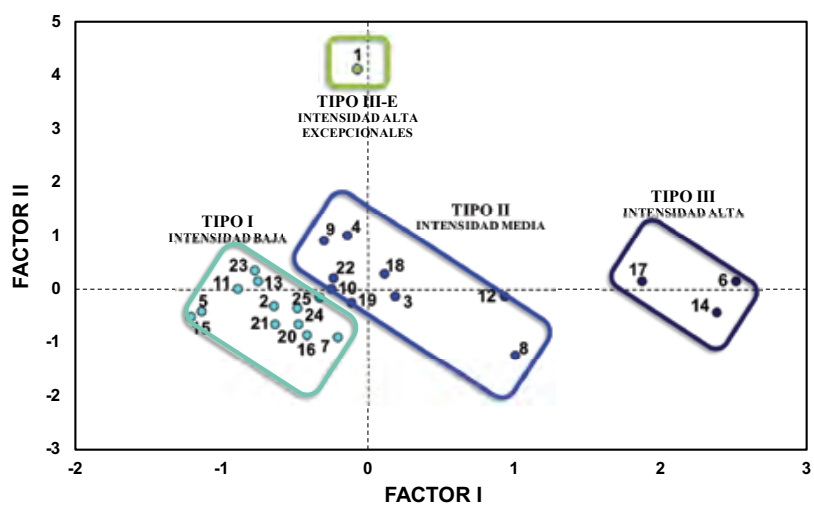

Figura 7. Distribución de los eventos de crecida en el plano factorial I-II obtenidos por el análisis de componentes principales

Fuente: elaboración propia. 
y con caudales no superiores a $10 \mathrm{~m}^{3} \mathrm{~s}^{-1}$. Tipo II: eventos de crecida de mediana intensidad; ocurrieron con menos frecuencia (36\%), con nueve eventos (3, 4, 8, 9, 10, 12, 18, 19 y 22). La contribución de SS se consideró altamente relevante $\left(100 \mathrm{t} \leq \mathrm{SS}_{\mathrm{t}} \leq 500 \mathrm{t}\right)$ y sus caudales máximos estuvieron comprendidos entre 10 y $21 \mathrm{~m}^{3} \mathrm{~s}^{-1}$. Tipo III: agrupa los eventos de mayor intensidad (1, 6, 14 y 17$)$ que representan el $16 \%$ del total analizado. Se caracterizan por una alta exportación de SS (> 500 t) y caudales máximos de importancia significativa (21 a $60 \mathrm{~m}^{3} \mathrm{~s}^{-1}$ ). Este tipo de evento se puede clasificar como excepcional debido a su intensidad y al tiempo relativamente corto que tarda en alcanzar el caudal máximo (Montoya, 2013).

Los resultados anteriores sugieren una respuesta directa de la cuenca en términos de transporte de sedimento en suspensión y carbono orgánico a eventos de gran importancia en términos de precipitación y volumen de agua generado. Los eventos de crecida de alta intensidad corresponden a aquellos con una alta capacidad para transportar SS, COD y COP. Esta clasificación contribuye a la comprensión de la cuenca en términos de transporte de contaminantes y la respuesta hidrológica de los eventos de crecida con diferente intensidad.

\section{CONCLUSIONES}

Es trascendental el monitoreo continuo de parámetros físicos, químicos, meteorológicos e hidrológicos para establecer la cantidad y variabilidad de sedimentos en suspensión y carbono orgánico que fueron exportados en la cuenca del río Oka.

La fase particulada en la columna de agua refleja una alta variabilidad en el transporte de los sólidos suspendidos en los eventos de crecida. La buena relación entre el caudal y la concentración de carbono orgánico disuelto, así como de la concentración de los sólidos suspendidos y la concentración de carbono orgánico particulado durante los eventos de crecida, fue útil para calcular las concentraciones a largo plazo a partir de parámetros simples de medir e hizo posible obtener la carga exportada por año.

Los resultados revelan el importante papel de las crecidas en la movilización de SS, COD y COP desde la cuenca agroforestal del Oka, contribuyendo a determinar cargas de 2267,5 t (SS), 104,2 t (COD) y 53,2 t (COP), que se corresponden con tasas de 71,85 t km² año-1 (SS), 3,30 t km² año (COD) y 1,68 t km-2 año-1 (COP), como valores medios para los tres años hidrológicos estudiados.

La precipitación y el volumen de agua transportado fueron los principales factores que controlaron la exportación de SS, COD y COP en la cuenca de cabecera del río Oka. Estos factores señalaron que las crecidas consiguen agruparse en tres categorías de acuerdo con la intensidad y su potencial para el transporte de sólidos en suspensión y carbono orgánico.

La metodología utilizada para llegar a establecer una clasificación de los eventos de crecida permite determinar la carga exportada por tipo de evento, mensual, y anual; las crecidas de intensidad superior fueron las de mayor importancia en la contribución de sólidos suspendidos y carbono orgánico a la salida de la cuenca. También proporciona información para un mejor diagnóstico del impacto de cada evento de crecida, contribuyendo a la proyección de normas y disposiciones para la protección del ecosistema fluvial y del estuario de Urdaibai.

\section{FINANCIAMIENTO}

Universidad del País Vasco (UFI11/26), Ministerio de Ciencia e Innovación (CGL2011-26236), Gobierno Vasco-Grupo Consolidado de Hidrogeología y Medio Ambiente (IT598-13).

\section{REFERENCIAS}

American Public Health Association (APHA) (2005). Standard methods for the examination of water and wastewater. 21a. ed. Washington, D. C. 
Carvajal, Y., Arango, D. y Jiménez, H. (2007). Estimación de caudales promedios mensuales por subcuencas hidrológicas mediante modelación con HEC-HMS. Tecnura, 11(21), 14-28. DOI: https:/ doi.org/10.14483/22487638.6260

Cerro, I., Sánchez-Pérez, J. M., Ruiz-Romera, E. y Antigüedad, I. (2014). Variability of particulate (SS, POC) and dissolved (DOC, NO3) matter during storm events in the Alegria agricultural watershed. Hydrological Processes, 28, 2855-2867. DOI: https://doi.org/10.1002/hyp.9850

Coynel, A., Etcheber, H., Abril, G., Maneux, E., Dumas, J. y Hurtrez, J. E. (2005). Contribution of small mountainous rivers to particulate organic carbon input in the Bay of Biscay. Biogeochemistry, 74, 151-171. DOI: https://doi.org/10.1007/s10533-004-3362-1

Diputación Foral de Gipuzkoa (DFG) (2012). Cuantificación de la tasa de sedimentos exportados de las cuencas del Territorio Histórico de Gipuzkoa. (Fase VI). Caracterización de los sedimentos en la cuenca del Deba. Año hidrológico 2011-2012, 2012. Informe inédito. Gipuzkoa, España.

González, N., Carvajal, Y. y Loaiza, W. (2016). Análisis de sequías meteorológicas para la cuenca del río Dagua, Valle del Cauca, Colombia. Tecnura, 20(48), 101-113. DOI: https://doi.org/10.14483/ udistrital.jour.tecnura.2016.2.a07

Ludwig, W., Probst, J. L. y Kempe, S. (1996). Predicting the oceanic input of organic carbon by continental erosion. Global Biogeochemical Cycles, 10, 23-41. DOI: https://doi.org/10.1029/95GB02925

Montoya, L.H. (2013). Efectos de las crecidas en el transporte de material particulado y contaminantes asociados: aplicación al caso del río Oka (Urdaibai), País Vasco. [Tesis inédita de doctorado]. Universidad del País Vasco, Bilbao, España.

Montoya, L.H., Ruiz, E. y Antigüedad, I. (noviembre, 2014). Efectos de las crecidas en el transporte de material particulado y carbono orgánico. Caso del río Oka (País Vasco, España). En Conferencia I Jornada Binacional de Investigación de la URBE (ClDETIU). Universidad Privada Dr. Rafael Belloso Chacín. Maracaibo, Venezuela.
Ni, H. G., Lu, F. H., Luo, X. L., Tian, H. Y. y Zeng, E. Y. (2008). Riverine inputs of total organic carbon and suspended particulate matter from the Pearl River Delta to the coastal ocean off South China. Marine Pollution Bulletin, 56, 1150-1157. DOI: https://doi. org/10.1016/j.marpolbul.2008.02.030

Oeurng, C., Sauvage, S. y Sánchez-Pérez, J. M. (2010). Dynamics of suspended sediment transport and yield in an intensive agricultural catchment in Southwest France. Earth Surface Processes and Landforms, 35, 1289-1301. DOI: https://doi.org/10.1002/ esp.1971

Oeurng, C., Sauvage, S., Coynel, A., Maneux, E., Etcheber, H. y Sánchez-Pérez, J.M. (2011). Fluvial transport of suspended sediment and organic carbon during flood events in a large catchment in southwest France. Hydrological Processes, 25(15), 2365-2378. DOI: https://doi.org/10.1002/hyp.7999

Richey, J. E., Hedges, J. I., Devol, A. H. y Quay, P. D. (1990). Biogeochemistry of carbon in the Amazon River. Limnology Oceanography, 35, 352-371. DOI: https://doi.org/10.4319/lo.1990.35.2.0352

Royer, T. y David, M. (2005). Export of dissolved organic carbon from agricultural streams in Illinois, USA. Aquatic Science. 67, 465-471. DOI: https://doi. org/10.1007/s00027-005-0781-6

Uriarte, A. (1998). Sediment dynamics on the inner continental shelf of the Basque Country (N. Spain). [Tesis inédita de doctorado]. Universidad de Southampton, Southampton, Inglaterra.

Veyssy, E., Etcheber, H., Lin, R., Buat-Ménard, P. y Maneux, E. (1998). Seasonal variation and origin of Particulate Organic Carbon in the lower Garonne River at La Re'ole (Southwestern France). Hydrobiologia, 391, 113-126. DOI: https://doi. org/10.1023/A:1003520907962

Walling, D. E. y Webb, B. W. (1985). Estimating the discharge of contaminants to coastal waters by rivers: some cautionary comments. Marine POIlution Bulletin, 16, 488-492. DOI: https://doi. org/10.1016/0025-326X(85)90382-0

Walling, D.E. y Webb, B.W. (1996). Erosion and sediment yield: a global overview. Erosion and 
sediment yield: global and regional perspectives. Proceedings of the Exeter Symposium. IAHS Publications, 236, 3-19. Recuperado de http://hydrologie.org/redbooks/a236/iahs_236_0003.pdf

Yang, Y., He, Z., Wang, Y., Fan, J., Liang, Z. y StoffeIla, P. J. (2013). Dissolved organic matter in relation to nutrients ( $\mathrm{N}$ and $\mathrm{P}$ ) and heavy metals in surface runoff water as affected by temporal variation and land uses-A case study from Indian River Area, south Florida, USA. Agricultural Water Management, 118, 38-49. DOI: https://doi.org/10.1016/j. agwat.2012.12.001

Zabaleta, A., Martínez, M., Uriarte, J. A. y Antigüedad, I. (2007). Factors controlling suspended sediments yield during runoff events in small headwater catchments of the Basque Country. Catena, 71(1), 179-190. DOI: https://doi.org/10.1016/j. catena.2006.06.007 